\title{
Directional effects on the reliability of non-axisymmetric support structures for offshore wind turbines under extreme wind and wave loadings
}

\section{Abstract:}

In comparison with monopile support structures commonly used for offshore wind turbines (OWTs) in shallow water, fixed bottom support structures for deeper water such as jackets tend to lack axisymmetry and have different capacities when loaded in different directions. Design of such a structure may therefore benefit from consideration of the directional characteristics of loading. This paper focuses on a rational and efficient approach to assess the structural safety of jacket type OWTs under directionally dependent extreme environmental loads. The Incremental Wind-Wave Analysis (IWWA) framework is reviewed and used to assess capacity of jacket-type OWTs under directional environmental wind-wave conditions. The approach uses static pushover analysis of OWT jackets subject to combined wind and wave load patterns corresponding to increasing mean return periods (MRPs). The wind and wave conditions are calculated independently and assumed to occur simultaneously. The loading direction and jacket orientation are both included in the analysis. To illustrate the approach, metocean conditions at different sites along the U.S. Atlantic coast are obtained from the historical database of the National Oceanic and Atmospheric Administration (NOAA) Data Buoy Center. Two models are introduced for estimating the occurrence probability of wave direction, one of which directly uses the frequency of wave directions and the other uses a Gaussian kernel to represent the range of wave directions represented by given directional spectra. For each combination of wind-wave direction and structural orientation, an IWWA analysis gives the capacity in terms of the MRP conditions leading to formation of a fully developed plastic mechanism in the jacket. Those capacities, convolved with MRP models for loading intensity, yield direction-dependent structural reliabilities, and when those reliabilities are convolved with the probability density function for loading direction, a total failure probability is obtained that accounts for wave directionality. Example analyses are conducted for an OWT supported by a four-leg jacket adapted from a design published as part of the European Union UpWind Project. Effects of extreme loading directionality, structural orientation, structural geometry and site specification on the ultimate capacity of the jacket are carefully discussed.

\section{Keywords:}

Wind energy; offshore wind turbine; support structure; directionality; reliability; structural orientation; optimization; safety assessment; incremental wind-wave analysis; extreme environmental conditions

\footnotetext{
${ }^{*}$ Corresponding author.

Address: 42D Marston Hall, University of Massachusetts Amherst, 130 Natural Resources Road, Amherst, MA 01003.

Tel: (+1) 413-577-1242. Email: kaiwei.tj@gmail.com
} 


\section{Introduction}

Renewable energy continues to contribute an increasing amount of energy to the global energy system and wind energy shows particular promise as a sustainable source of energy. It is planned that $20.0 \%$ of the electricity demand of the United States and $28.5 \%$ of the demand of the European Union will come from wind energy by 2030 [1,2]. The efficiency of offshore wind energy extraction will play a significant role in meeting these wind energy targets. Construction of offshore wind turbines (OWTs) requires different types of support structures for different water depths and geotechnical conditions, e.g. monopiles for shallow water up to $25-30 \mathrm{~m}$, jackets and tripods for deep water (30 - 80m depth) and floating platforms for water deeper than $80 \mathrm{~m}$. Continued development of design and risk assessment techniques for such structures can play an important role in continuing to lower the overall cost of energy for offshore wind since the support structure can account for approximately $25 \%$ of the initial capital cost of an OWT [3].

Most of the support structure types, except monopiles, are not axisymmetric. For example, a typical OWT jacket has 3 or 4 legs and a triangular or square cross-section. These kinds of nonaxisymmetric support structures have different stiffness and capacity when loaded in different directions. The American Petroleum Institute (API) suggests that a minimum of 8 directional analyses are required for symmetrical, rectangular and square platforms, a minimum of 12 directions are required for tripod jackets, and even more additional directions are required for other unsymmetrical structures to ensure appropriate structural safety [4]. Further directional dependence comes from the fact that environmental loading intensity (e.g. wind speed and wave height) also varies with direction. Previous studies that assume wind and waves are unidirectional and load the support structure from a deterministic direction clearly cannot fully account for the directional dependence of capacity and loading that will influence the reliability of non-axisymmetric OWT support structures $[5,6]$. Neglecting the effect of directionality in the analyses of OWT support structures [7, 8] surely does not allow for optimization of the structural orientation to minimize overall failure probability. In principal, it is possible that the structural orientation corresponding to minimum failure probability may not coincide with that which aligns the structural orientation with greatest capacity in the direction of most likely loading. Furthermore, it should be noted that the orientation of the support structure is essentially a free parameter in the design since the turbine is free to yaw and can face into the prevailing wind to maximize energy generation regardless of structural orientation. Therefore, a framework including the metocean conditions with directional dependences is valuable for risk and safety assessment of non-axisymmetric OWT support structures.

The effect of loading directionality on the operational response, ultimate capacity, fatigue performance, natural frequencies, etc. of offshore support structures has been the subject of some attention in the open literatures. Haver [9] studied the effect of wave directionality both for the quasi-static response and for the dynamic response of offshore jackets and stated that the extreme response is very sensitive to the platform orientation when all the sea states are assumed to approach from one direction. Bea et al. [10] highlighted the importance of the directional characteristics of the waves on the magnitude of measured deck forces, a loading that has been cited as the main reason for localized damage to the superstructure of oil platforms during hurricanes [11]. Li [12] further studied the directional effect of wind and wave loads on OWT jackets using a quasi-static pushover procedure and indicated that (i) the direction of the wind and wave force affects the structure's failure mechanism and ultimate strength significantly; (ii) the largest capacity of the jacket is attained when the jacket is oriented broadside to the dominant 


\section{3}

\section{4}

wave direction and the minimum capacity is attained when waves approach at 45 degrees to the sides of a square plan jacket. Philippe et al. [13] found through modal analysis that natural modes for a particular floating OWT system are excited differently depending on the approaching direction of the waves. Mittendorf et al. [14] investigated the influence of directional irregular wave models on damage equivalent fatigue loads of OWTs and found that the consideration of the waves' directionality results in an approximate reduction of the observed fatigue loads of up to $20 \%$ compared to unidirectional models.

Many approaches have been developed to obtain directional environmental loading for analysis and design of non-axisymmetric offshore structures. One approach is specified in the API code [4] and recommends a wave height adjustment factor to account for the wave directionality in the Gulf of Mexico without the use of a directional and irregular nonlinear design wave. This is a simplified approach that allows an analysis to account for directional effects [15], but the development of the adjustment factors requires lengthy metocean monitoring and does not supply any probability information of the direction of approaching waves. A second approach to directional analysis is to generate a stochastic wave simulation that includes multidirectional waves corresponding to a directional wave spectrum [9, 16-18]. This approach depends on the directional wave spectrum, which is always site-specific [19], and requires dynamic time history analysis which can be prohibitively time consuming when structural nonlinearities are to be included as well. A third approach to directional analysis borrows from both approaches and attempts to estimate possible directional extremes based on criteria obtained from existing sources of observed or simulated directional wave statistics [20,21]. Our study is based on the third type of approach to assess the directional intensities of OWT support structures.

This paper adds to the literature on directional effects for offshore structures by: using Incremental Wind-Wave Analysis (IWWA) [22] to assess structural capacity in a more accurate load-pattern dependent way; assessing directional effects for a realistic OWT jacket support structure; illustrating the approach with wave direction distributions and wind/wave intensity distributions derived from measured data from the U.S. coast; convolving wind/wave direction and intensity distributions to assess structural reliability as a function of jacket orientation.

\section{Analysis Framework}

\subsection{General configurations and assumptions}

Figure 1 (a) shows the general configuration of a jacket-supported OWT that consists of the entire OWT assembly up to the bottom of the rotor-nacelle assembly (RNA). $\theta_{\text {load }}$ here refers in particular to the dominant wave direction, which dominates the failure of OWT jacket [22]. Since the jacket is a wave dominated structure (in the absence of yaw error) the wind is treated here as co-directional with the wave field. The structural configuration of the jacket is based on the model jacket defined in the European Union UpWind report [23], and the tower and RNA correspond to the widely disseminated National Renewable Energy Laboratory (NREL) 5-MW turbine [24]. Several simplifying assumptions regarding the loading conditions are made and these allow primary attention to be paid to the role of loading directionality in governing the response of jacket-supported OWTs to extreme loading. First, the wind and wave loads are assumed to be co-directional without wind-wave misalignment. Second, perfect yaw control is 

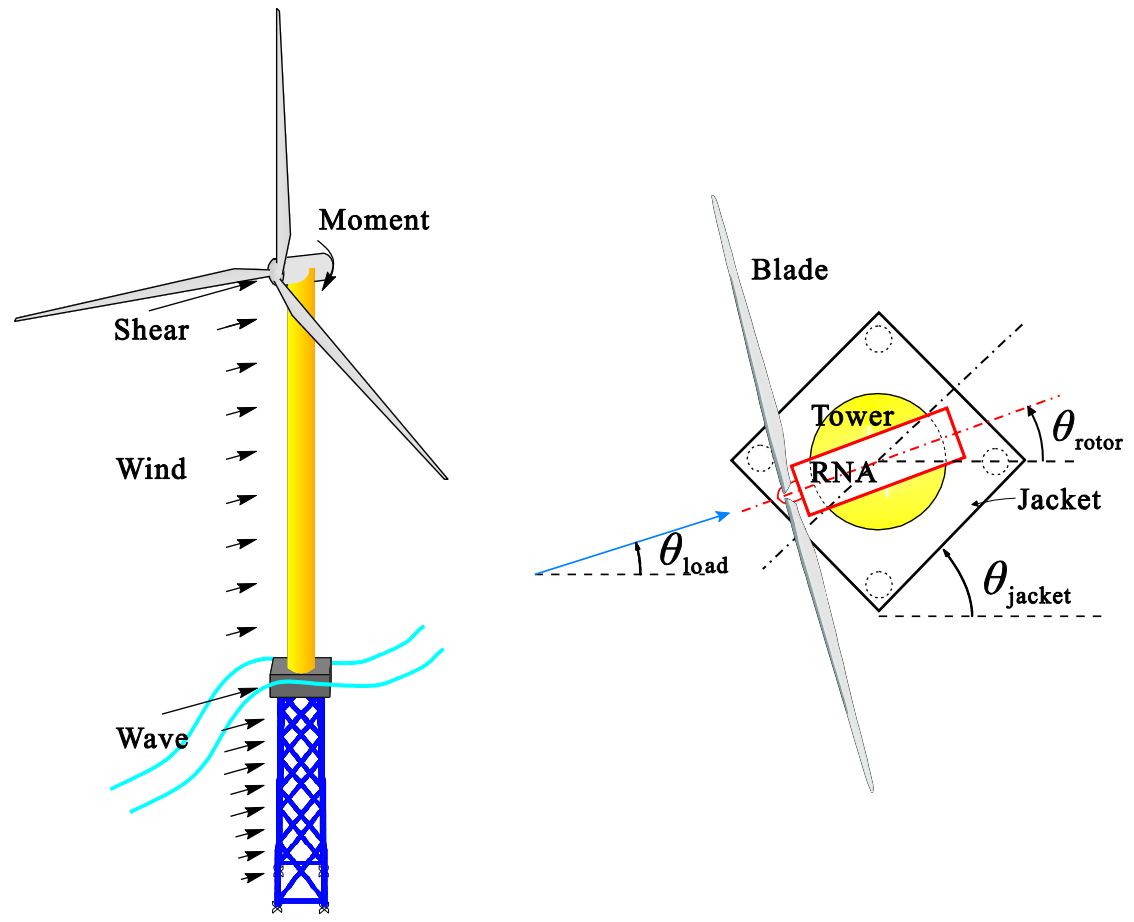

(a) Support structures

(b) Structural orientation and loading direction static approach to evaluate the capacity of OWT support structures subject to arbitrary combinations of wind and wave load. The approach, referred to as the IWWA1 procedure [22], combines independently evaluated wind and wave conditions at common mean return periods (MRPs). This approach neglects the correlation between wind and wave conditions and assumes that the MRPs of the wind and wave conditions are independent and the wind and wave conditions at equivalent MRPs occur simultaneously. Industry standards for offshore structures such as those published by API [4] and IEC [25] prescribe methods for determining wind and wave conditions from independent distributions. However, many papers have studied the effect of wind and wave correlation through FORM (First Order Reliability Method) [26] and IFORM (Inverse First Order Reliability Method) [27-29] and have highlighted that the assumption of independence is conservative and unrealistic under actual offshore conditions. The IWWA framework includes another approach, referred to as the IWWA2 procedure [22], which uses joint probability models to estimate wind speed and wave height at design return periods. A case study of the IWWA approach for a jacket-supported OWT located off the U.S. Atlantic coast [22] showed that the difference in the reliability index corresponding to probability of failure (i.e., when a full plastic mechanism forms in the jacket) over twenty years is less than $2 \%$ when comparing probabilities calculated based on the IWWA1 procedure and based on the IWWA2 procedure. This small difference is due to the dominance of wave loads relative to wind loads on the jacket during extreme conditions when the turbine is not operational with no yaw error and 
investigated or a different structural configuration with more equal wind and wave effects were to be investigated, a larger difference between the IWWA1 and IWWA2 results would be expected. Because the conditions considered in this paper are similar to that in the referenced case study [22] and to keep the paper focused on directional effects, the IWWA1 procedure is used in this paper.

157 Dynamic effects of wind and wave loads, such as wind turbulence, wave irregularity, windwave-structure interaction, inertial loads, time-dependent variance of loading direction and amplitude, etc., are neglected by the static IWWA approach considered here. The influence of dynamic effects has been addressed by Golafshani et al. [30] for offshore oil and gas support structures and they found that, for two different example platforms, the difference between the dynamic and static results was either negligible (less than $0.5 \%$ ) or approximately $14 \%$ and that the static analyses provided a conservative estimate of capacity. The difference between the two examples is due to differences in the dynamic behavior of the platform. Although OWTs are more flexible than $\mathrm{O} / \mathrm{G}$ platforms and thus may be more susceptible to dynamic effects, the objectives of this paper are to isolate the effects of and provide initial insight into the directional dependence of OWT support structural capacity and reliability. For these reasons, combined with the large computational demands and additional complications of structural evaluation methods based on dynamic time history analyses, dynamic effects are ignored here to keep the paper focused on the directional effects.

\subsection{Directional IWWA analysis}

172 Consider an OWT support structure with an orientation of $\theta_{\text {jacket }}$ and a loading direction of $\theta_{\text {load }}$.

173 These two parameters completely define the incident direction of wind and wave loading since 174 wind-wave misalignment is assumed to be zero. In the presence of wind and wave intensities that 175 are independent of direction, a single parameter $\left|\theta_{\text {jacket }}-\theta_{\text {load }}\right|$ would suffice for parameterizing

176 the directional analysis. Although such an assumption regarding directional dependence of wind and wave intensity is made in this paper, the two parameter characterization is retained in the definition of the approach to allow generalization to the case in which wind and wave intensities vary with direction. The directional IWWA approach introduced here involves probability models for $\theta_{\text {load }}$ and for the intensity of the loading. The probability density function (PDF), which defines the variability in loading direction, can be estimated from data in a variety of ways, two of which are described later in this paper. The probability model for the loading intensity consists of generalized extreme value distributions for the annual maxima of the wind speed and wave height $f_{V_{w}}\left(v, \theta_{\text {load }}\right)$ and $f_{H_{s}}\left(h, \theta_{\text {load }}\right)$. From these models, which are fit to data as described in following sections, the value of the wind speed and wave height at given values of the MRP can be obtained. The MRP is then used as a single parameter characterization of the aligned wind and wave loading. It should be noted that in principle other methods for parameterizing the loading intensity, such as the inverse first order reliability method (IFORM) [27-29], could be used within the directional IWWA context, and that independent models for the wind and wave intensity are used here to simplify the analysis and presentation of directional effects and keep 191 the paper focused on the directionality.

192 The loading intensities and directions are converted to structural load effects using a structural

193 model. Once this model has been developed along with probabilistic models for the loading 
194 direction and intensity, the following procedure is performed to complete the directional IWWA 195 analysis.

196 1. Discretize the range of directions $\left[0^{\circ}, 360^{\circ}\right]$ into $n_{\theta}$ even intervals with lower and upper boundary values among a periodic series of $\left\{\frac{\Delta \theta}{2}, \frac{3 \Delta \theta}{2}, \ldots, \frac{\left(2 n_{\theta}-1\right) \Delta \theta}{2}\right\}$, where $n_{\theta} \Delta \theta=360^{\circ}$

2. Set $\theta_{\text {jacket }}=\theta_{\text {jacket }, i}, i \in\left[1, n_{\theta}\right]$

3. Set $\theta_{\text {load }}=\theta_{\text {load }, j}, j \in\left[1, n_{\theta}\right]$

4. Perform a single parameter IWWA [22] to determine the MRP corresponding to failure of the support structure with an orientation $\theta_{\text {jacket }}=\theta_{\text {jacket, } i}$ under wind and wave loading approaching from $\theta_{\text {load }}=\theta_{\text {load }, j}$. Note that the IWWA provides the MRP value at which the wind and wave loads lead to the formation of a fully developed mechanism in the structure and accounts for variation of the load pattern as wave height and wind speed intensities increase. The result of the IWWA gives $\mathrm{MRP}_{\text {fail }}\left(\theta_{\text {jacket }}, \theta_{\text {load }}\right)$, the MRP corresponding to failure as a function of the structural orientation and loading direction.

5. Repeat from step 3, incrementing the value of $j$ so that all load directions are considered.

6. Repeat from step 2, incrementing the value of $i$ so that all structural orientations are considered.

211 Once the IWWA curve and corresponding values of $\mathrm{MRP}_{\text {fail }}\left(\theta_{\text {jacket }}, \theta_{\text {load }}\right)$ are obtained, the annual failure probability at a given value of $\theta_{\text {jacket }}$ is

$$
P_{f, 1}\left(\theta_{\text {jacket }}\right)=\int_{0}^{360} P_{f, 1}\left(\theta_{\text {jacket }}, \theta_{\text {load }}\right) \cdot f_{\theta_{\text {load }}}(\theta) d \theta_{\text {load }}
$$

214 in which

$$
P_{f, 1}\left(\theta_{\text {jacket }}, \theta_{\text {load }}\right) \approx \frac{1}{M R P_{\text {fail }}\left(\theta_{\text {jacket }}, \theta_{\text {load }}\right)}
$$

is an approximation to the annual failure probability that is widely considered accurate for $M R P$ $>10$ years and is obtained directly from the directional IWWA curve. Given the annual failure probability at a specified value of $\theta_{\text {jacket }}$ the failure probability over a specified design lifetime of

219 length $n_{\text {years }}$ is

$$
P_{f, n_{\text {years }}}\left(\theta_{\text {jacket }}\right)=1-\left(1-P_{f, 1}\left(\theta_{\text {jacket }}\right)\right)^{n_{\text {years }}}
$$

221 and the problem of selecting an optimal orientation $\hat{\theta}_{\text {jacket }}$ for the support structure can be cast as 222 the minimization problem

223

$$
\hat{\theta}_{\text {jacket }}=\arg \min _{\theta_{\text {jacket }}}\left(P_{f, n_{\text {years }}}\left(\theta_{\text {jacket }}\right), \theta_{\text {jacket }}\right)
$$




\section{Definition of probabilistic models}

\subsection{Occurrence probability of dominant wave directions}

Historical measurements of ocean wave conditions can be used to calibrate the PDF of the direction of the wave with maximum power spectral density, which is needed for a directional IWWA, and such data usually take the form of a directional wave spectrum $S(f, \theta)$ that give the power associated with the wave field for a range of incident directions $\theta$ and frequencies $f$ $[31,32]$. In the absence of historical measurements, ocean wave modeling and numerical simulations are useful alternatives for calibrating wave direction PDFs [19, 33]. Although the direction of the wave with maximum power spectral density $\left(\theta_{\text {load }}\right.$ since the wind and wave are assumed to be co-directional) is a continuous random variable bounded by the domain $\left[0,360^{\circ}\right]$, calibration of $f_{\theta_{\text {load }}}(\theta)$ will usually rely on a finite set of $n_{\text {obs }}$ observations of $\theta_{\text {load }}$, for example, the historical hourly meteorological database of National Data Buoy Center (NDBC) [34, 35], a part of U.S. National Oceanic and Atmospheric Administration (NOAA).

\subsubsection{Frequency-based PDF}

In the NDBC database, the dominant wave direction (MWD), where the waves with the maximum energy come from, has been continuously recorded for the past several years in the form of the peak direction of spectra obtained from hourly measurements of the wave field. Therefore, the range of wave directions is discretized into $n_{\theta}$ even intervals of $\Delta \theta$, and a direct estimate of the occurrence probability $f_{\theta_{\text {toad }}}(\theta)$ of wave directions in the interval $\left[\theta_{j}, \theta_{j}+\Delta \theta\right]$ can be counted by

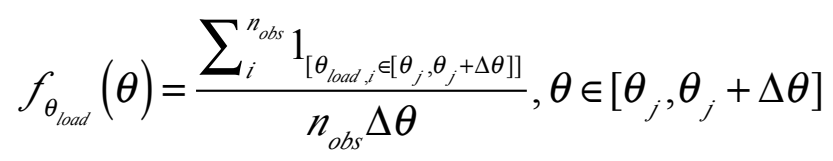

where $1_{[.]}$is an indicator variable, which equals 1 if $\theta_{\text {load }}$ falls in the domain $\left[\theta_{j}, \theta_{j}+\Delta \theta\right]$ and 0 otherwise. This approach essentially approximates the continuous wave direction PDF by the histogram of dominant wave directions obtained from data normalized to have the same dimensions as a PDF. In effect, this approach assigns the entirety of the probability mass from a given observation to the dominant wave direction measured during the observation. In the case where $n_{o b s}$ is relatively small, such an approach may result in a PDF for wave direction that lacks smoothness typical of PDFs of continuous random variables appearing in physical processes. Moreover, this approach neglects the spreading of the wave energy over a range of directions around the dominant wave direction (MWD).

\subsubsection{Gaussian-kernel-smoothed PDF}

An alternative approach to approximating $f_{\theta_{\text {toad }}}(\theta)$ considers the full content of the directional wave spectrum $S(f, \theta)$. Figure 2 (a) shows an example directional wave spectrum derived from NDBC measurements that illustrates the spread of wave power over a broad range of directions (approximately $\pm 75^{\circ}$ from the dominant wave direction) but over a relatively narrow band of frequencies. Figure 2 (b) shows $S\left(f_{\text {dom }}, \theta\right)$, a circumferential section through the directional 
spectrum at the frequency $f_{\text {dom }}$ corresponding to peak power. This figure clearly shows the spread of the wave power over a range of directions centered at the dominant wave direction. Note that the regions of negative power are numerical artifacts of the procedure used to reconstruct the directional spectrum from parameterized data provided, in this case, by NDBC $[34,35]$. In this alternative approach to approximating $f_{\theta_{\text {toad }}}(\theta)$, it is assumed that the spread of the directional spectrum indicates the possibility that the extreme wave might come from a direction other than the dominant wave direction. This is accomplished by fitting a kernel function to $S\left(f_{\text {dom }}, \theta\right)$ and then summing and normalizing such a kernel for each of the observations. The result of this operation is to spread the wave direction PDF over a broader range of directions. In the absence of higher frequency wave direction and amplitude data that would allow direct estimation of $f_{\theta_{\text {lood }}}(\theta)$ it is not possible to assess which of these approximations provides a better view of the actual wave field. Both are presented here to illustrate the range of possible effects. The kernel-based procedure is:

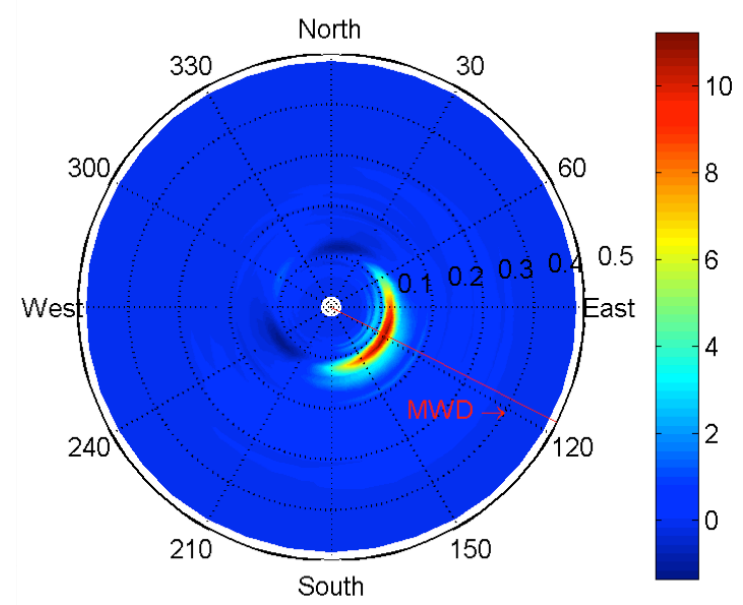

(a) Directional wave spectrum $\left(\mathrm{m}^{2} / \mathrm{Hz} \mathrm{deg}\right)$

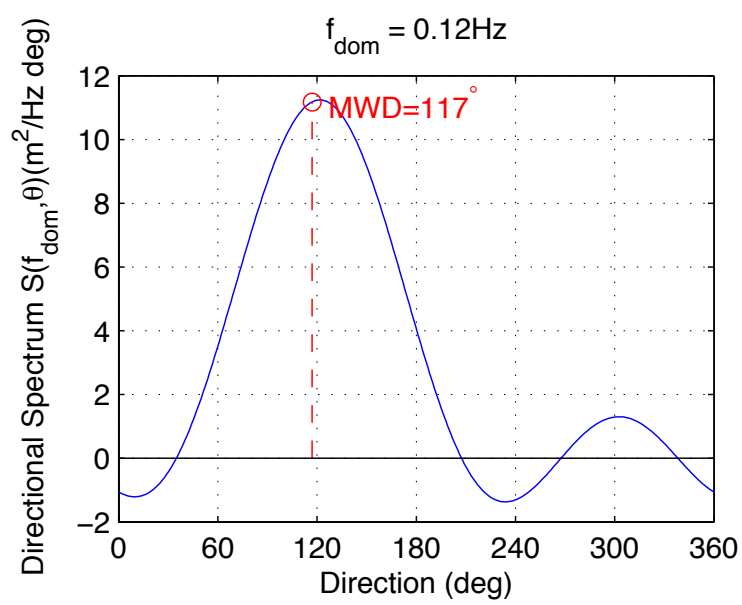

(b) Circumferential section through directional wave spectrum at dominant frequency

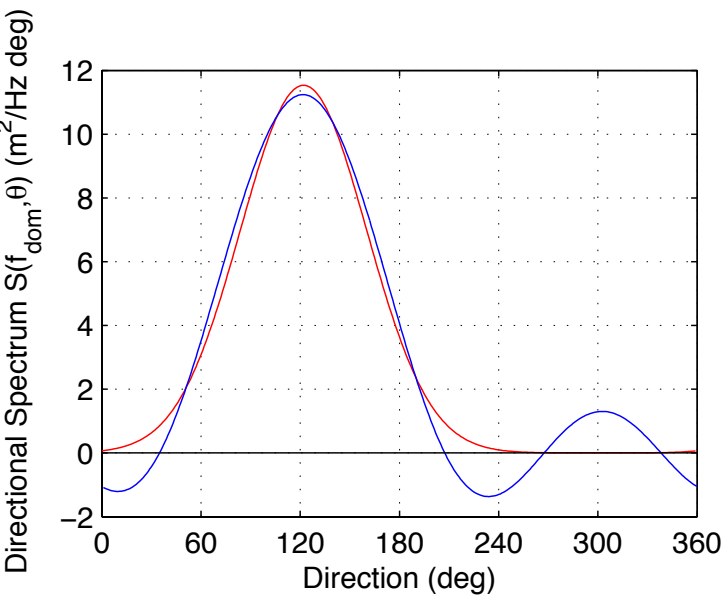

(c) Best fit Gaussian kernel to directional spectrum of (b) 
277

278

279

280

281

282

283

284

285

286

287

Figure 2 Procedure of converting directional spectrum information into a smoothed probability model for wave direction

1. Select an observation and extract $S\left(f_{\text {dom }}, \theta\right)$, the spectral power as a function of direction at the dominant wave frequency.

2. Filter the $S\left(f_{\text {dom }}, \theta\right)$ according to

$$
\hat{S}\left(f_{\text {dom }}, \theta\right)=\left\{\begin{array}{cl}
S\left(f_{\text {dom }}, \theta\right), & S\left(f_{\text {dom }}, \theta\right) \geq 0 \\
0 & S\left(f_{\text {dom }}, \theta\right)<0
\end{array}\right.
$$

and remove negative value artifacts in the directional spectrum.

3. Fit a Gaussian kernel $S_{\text {gauss }}\left(f_{\text {dom }}, \theta\right)$ to $\hat{S}\left(f_{\text {dom }}, \theta\right)$ (Figure $2(\mathrm{c})$ ).

4. Repeat steps 1-3 for $n_{o b s}$ observations of the directional spectrum.

5. Define the smoothed wave direction PDF as

$$
f_{\theta_{\text {load }}}(\theta)=\frac{1}{n_{\text {obs }}} \sum_{i=1}^{n_{\text {obs }}} S_{\text {gauss }}\left(f_{\text {dom }}, \theta\right)
$$

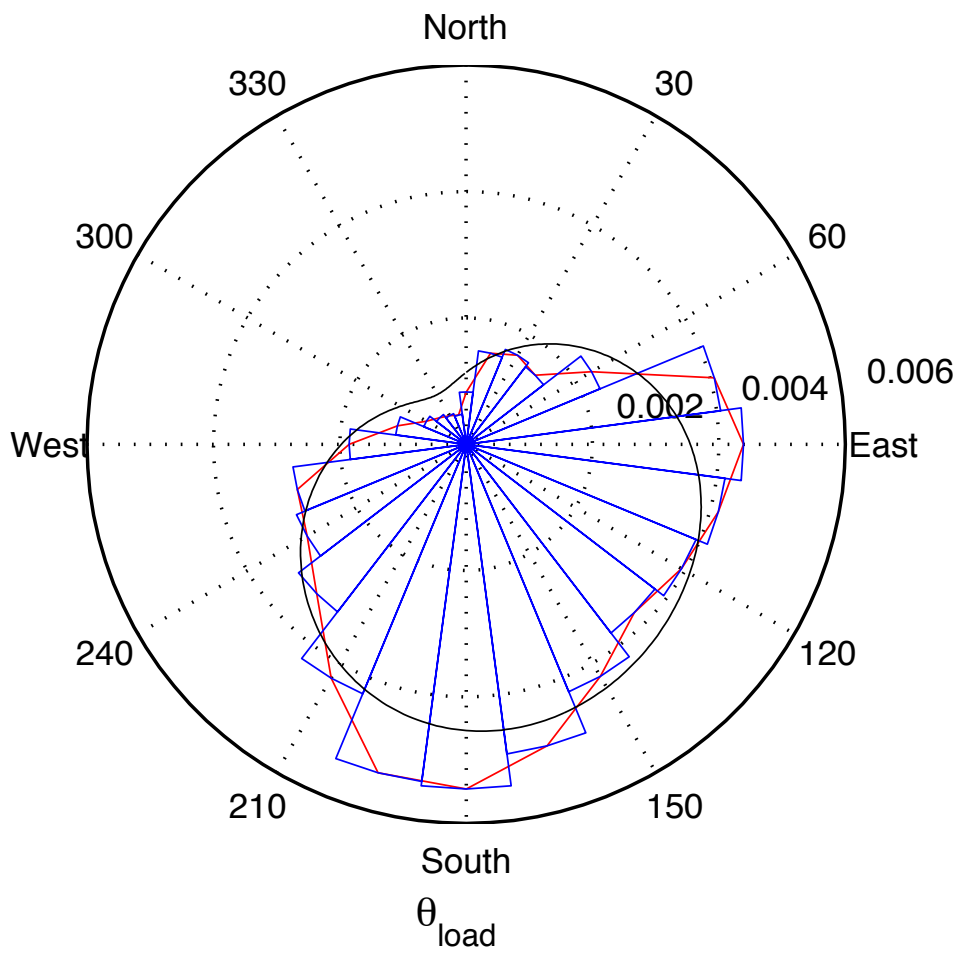

Figure 3 Probability density function (PDF) of most likely wave direction from measurement of Buoy 44008 off the coast of Massachusetts (red line is PDF based on the histogram of observed dominant wave direction, i.e. the direct frequency approach; black line is smoothed PDF based on the Gaussian kernel approach)

Figure 3 shows an example wave direction PDF $f_{\theta_{\text {load }}}(\theta)$ for a site off the coast of Massachusetts (MA; Station 44008) as estimated from the direct frequency based approach of Eq. 
5 and the smoothed, Gaussian kernel approach of Eq. 7. The use of the Gaussian kernel

296 significantly alters $f_{\theta_{\text {load }}}(\theta)$, lowering peak probability density values near $180^{\circ}$ (South) and

297 adding probability mass in regions with few occurrences of the dominant wave direction near

$298330^{\circ}$ (North-northwest).

\subsection{Probability model of extreme wind and wave conditions}

300 The single parameter IWWA used in the directional analysis presented here uses the MRP to 301 characterize the intensity of the wind and wave loading. The wind condition is measured by a 1 minute average at a hub height of $90 \mathrm{~m}$, denoted by $V_{w}$ and the wave condition is measured by the extreme wave, denoted $H_{e}$ and taken to be $1.86 H_{s}$ where $H_{s}$ is the significant wave height [25]. Data from environmental measurements are used to obtain a set of annual maxima of $V_{w}$ and $H_{e}$. For each of $V_{w}$ and $H_{e}$ a generalized extreme value distribution [36] is fit to the set of annual maxima and the wind speed and wave height corresponding to a given MRP are extracted from the upper tail of the distribution by

$$
V_{w, M R P}=F_{V_{w}}^{-1}\left(1-\frac{1}{M R P}\right)
$$

with $H_{s}$ substituted for $V_{w}$ as appropriate. Equation 8 applies an approximation that $\frac{1}{M R P}$ is taken to be the annual probability of exceedance, which is valid as long as the mean return period is relatively long. It should be emphasized that the annual maxima approach described here considers some very long MRPs, up to hundreds of thousands of years. Such values are not generally used in the engineering design and are unreliable to estimate, lying far into the upper tail of the distribution. However, such events can have an important contribution to the total failure probability, which is needed by decision makers regarding the risk profile of the structure and attendant financing. It is also worth mentioning that attempting to estimate extreme wind speeds and wave heights from a limited duration of measurements of annual maxima may underestimate the severity of the conditions, particularly for locations exposed to hurricanes.

\section{Example jacket and environmental data}

The procedure described in sections 2 and 3 for assessing directional reliability of OWT jackets using the IWWA approach will be illustrated with a numerical example representative of U.S. Atlantic coast sites with the potential to experience OWT development in the coming years and decades. In this section the jacket support structure used in the example is described along with the corresponding structural model as are the source and characteristics of the environmental data use in the example.

\subsection{Load and structure modeling}

The non-axisymmetric OWT support structure investigated here is a jacket support structure developed to support the NREL 5-MW turbine [24]. The design was made as part of the UpWind project of the European Union [23] to serve as a research and development tool. As shown in Figure 4, the hub height is $90 \mathrm{~m}$ above the mean sea level. The nacelle has a diameter of $3 \mathrm{~m}$ and a total mass of $240,000 \mathrm{Kg}$. The three-bladed rotor has a diameter of $126 \mathrm{~m}$ and a mass of $110,000 \mathrm{Kg}$. The jacket is rotationally symmetric with a period of $90^{\circ}$ and consists of four legs and four levels of X-braces and cross braces. The top and bottom widths of the jacket are $8 \mathrm{~m}$ 
and $12 \mathrm{~m}$, respectively. A reinforced concrete deck with a mass of $666,000 \mathrm{Kg}$ and plan dimensions of $4.0 \times 9.6 \times 9.6 \mathrm{~m}$ is positioned on top of the jacket as the transition piece or platform connecting the jacket with the OWT tower. The jacket is assumed to be fully fixed at the mudline. The Young's modulus of the jacket steel is $210 \mathrm{GPa}$, the yield stress is $345 \mathrm{MPa}$ and the ultimate stress is $510 \mathrm{GPa}$ at a strain of 0.11 . The density is $8,500 \mathrm{Kg} / \mathrm{m}^{3}$, higher than the density of steel to account for paint, bolts, welds and all other additional masses that are not otherwise considered.

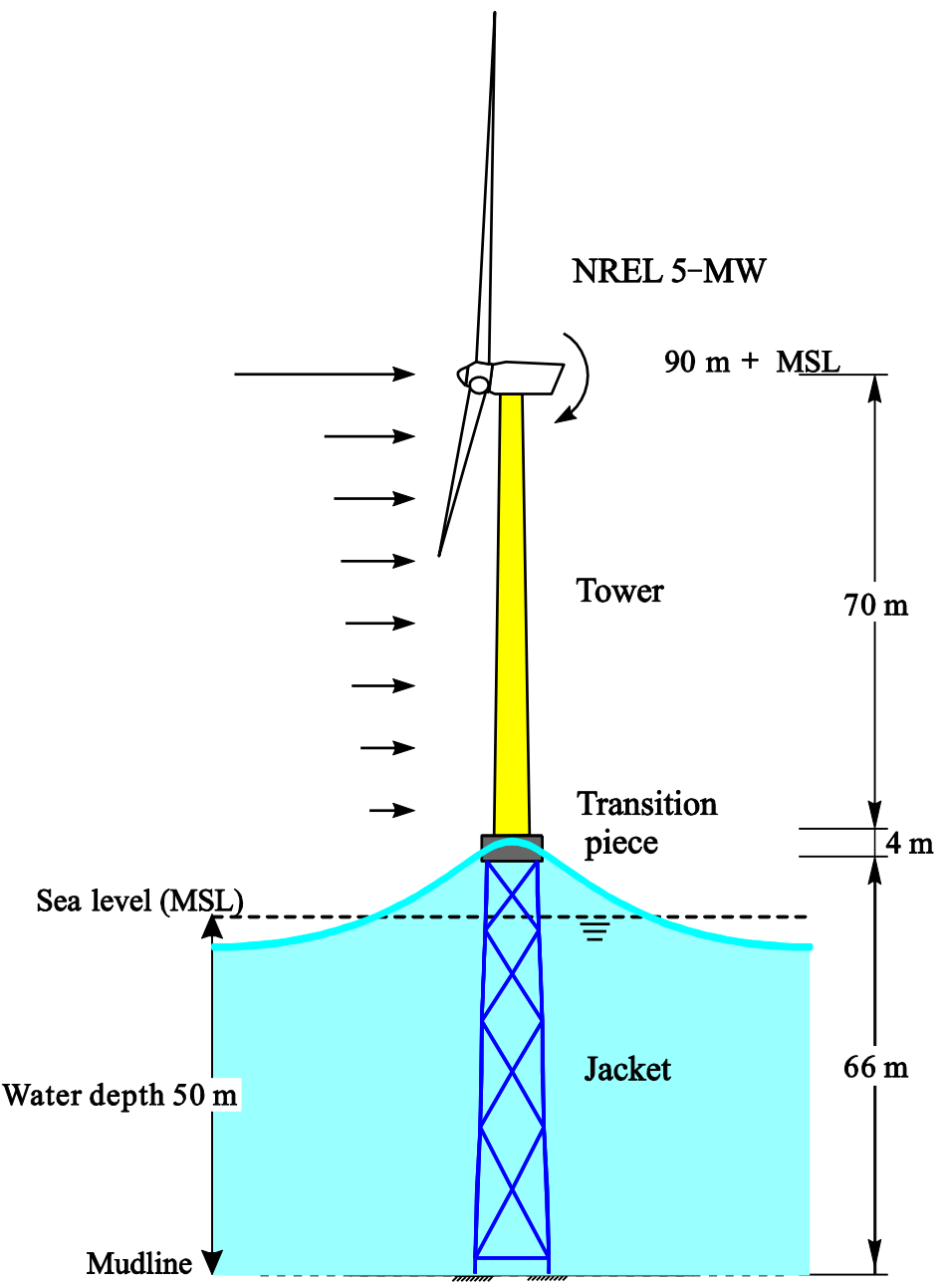

Figure 4 Elevation view of the UpWind jacket and OWT

The IWWA framework requires a static nonlinear pushover analysis of the jacket structure under wind and wave loading corresponding to a given MRP. Once a value of $V_{w, M R P}$ has been extracted from $F_{V w}(v)$, the aerodynamic forces on the OWT are determined with the aid of the computer-aided engineering tool FAST [37]. The aerodynamic loads on the rotor are calculated based on a steady wind with magnitude equal to $V_{w, M R P}$ and the aerodynamic loads on the tower are calculated according to the recommendation of the DNV specification [38]. The wind speed is assumed to vary with height above sea level according to power law with a wind shear exponent of 0.14 [25].

Hydrodynamic loads on the submerged part of the jacket are calculated by using nonlinear stream function and Morison's equation. Wave force on the jacket is always drag dominated due 
to the slender member dimensions. Stream function theory provides the water particle velocities and accelerations at the crest of the wave, which is the location of peak force for drag-dominated jacket structures, and Morison's equation [39] is then used to calculate three dimensional inertial and drag forces on each submerged member of the jacket. For extreme environmental conditions when the wave height is such that the wave crest contacts the deck of the jacket, a Morison-type approach is used to calculate the so-called wave-in-deck force generated by this interaction [4].

The jacket structure is discretized into three-dimensional beam elements in the commercial finite element package SAP2000 [40] with eight elements used for each structural member. Joints are assumed to be rigid. Geometric and material nonlinearity is included in the model, with material nonlinearity being included through a fully interacting three dimensional axial force - bending moment plastic hinge model.

\subsection{Environmental (wind and wave) data}

365

366

367

368

369

370

Selection of example sites for illustration of the directional IWWA approach is guided by many factors including: the extensive wind resource present off the Atlantic coast of the U.S., the water depth of $50 \mathrm{~m}$ that is assumed in the design of the example jacket structure used here, the desire to represent a broad range of directional characteristics of the wind and wave conditions, and the need to select sites for which there is a sufficient database of metocean conditions to estimate the wave direction PDF $f_{\theta_{\text {load }}}(\theta)$ and the extreme value distributions $f_{V_{w}}(v, \theta)$ and $f_{H_{s}}(h, \theta)$. Three sites have been selected to serve as illustrations in this study and they are listed, along with their basic characteristics, in Table 1.

It is important to note that the water depths vary considerably among the three sites while the jacket studied here was designed for a $50 \mathrm{~m}$ water depth. While it would have been preferable to identify several sites with similar water depth, there was no such availability in the NOAA database. To address this inconsistency, the wave data are modified in the following ways to make them more appropriate for a site with $50 \mathrm{~m}$ water depth:

1. For the MA site the water depth exceeds the $50 \mathrm{~m}$ design depth of the example jacket and the NOAA wave data are not adjusted. In principle this could result in artificially high wave heights being imposed on the example jacket, but in the authors' experience, wave height is not likely to differ significantly for sites with $50 \mathrm{~m}$ and deeper water depth exposed to similar wave generating conditions. Therefore the NOAA data are used directly.

2. For the $\mathrm{DE}$ and $\mathrm{ME}$ sites, the water depth is much smaller than the $50 \mathrm{~m}$ design water depth of the example jacket structure. Therefore the NOAA wave data are used only to estimate the wave direction PDF $f_{\theta_{\text {toad }}}(\theta)$ and the wave height from the MA site is used. This results in fictitious conditions at the DE and ME sites. Essentially we assume that sites exists that combine the directional properties of the DE and ME sites with the wave height properties of the MA site. In the absence of other NOAA buoys with water depths consistent with the jacket structure used here, this hybrid approach has been used to allow numerical examples of the assessment of jacket response to different directional characteristics.

Although this approach to using the wave conditions at three different sites with three different water depths is admittedly somewhat artificial, there simply are not, to the authors' knowledge, 
historical wave data at multiple Atlantic coast sites that meet all the criteria for use as examples in this paper and the adjustments described above have been made to allow examination of sitespecificity in the directional IWWA results.

Table 1 Information of NDBC buoy stations

\begin{tabular}{ccccccc}
\hline $\begin{array}{c}\text { Station } \\
\text { ID }\end{array}$ & Station Name & Lat / Long & $\begin{array}{c}\text { Distance } \\
\text { to shore } \\
(\mathrm{km})\end{array}$ & $\begin{array}{c}\text { Water } \\
\text { depth } \\
(\mathrm{m})\end{array}$ & $\begin{array}{c}H_{\mathrm{s}} \text { Record } \\
\text { length } \\
\text { (year) }\end{array}$ & $\begin{array}{c}\theta_{\text {load }} \text { Record } \\
\text { length } \\
\text { (year) }\end{array}$ \\
\hline 44007 & Maine (ME) & $43.531^{\circ} /-70.144^{\circ}$ & 6 & 23.7 & 32 & 5 \\
44008 & Massachusetts (MA) & $40.502^{\circ} /-69.247^{\circ}$ & 160 & 64.8 & 32 & 7 \\
44009 & Delaware (DE) & $38.461^{\circ} /-74.703^{\circ}$ & 32 & 30.5 & 28 & 1 \\
\hline
\end{tabular}

\section{Directional characteristics of environmental conditions}

This section describes analyses of the environmental data collected from the NDBC for the Maine, Massachusetts, and Delaware sites. The assumption that wind speed and wave height are co-directional means that the analysis of directionality presented here focuses solely on the wave direction which is the dominant loading for the jacket structure. In most of the directional results presented, the range of $\theta$ has been discretized into a series of $15^{\circ}$ intervals $I_{\theta i}=\left[\theta_{i}-7.5^{\circ}, \theta_{i}+7.5^{\circ}\right.$ ), $\mathrm{i}=1, \ldots, 24$ with the interval midpoints given by $\theta_{i}=15^{\circ}(\mathrm{i}-1)$. This discretization is made in order to allow estimation of the continuous functions $f_{\theta_{\text {load }}}(\theta)$ and $f_{H_{s}}(h, \theta)$ from the discrete NOAA data set. The wave height function has here been assumed independent of direction in the absence of data strongly indicating otherwise.

\subsection{Wave direction PDF}

Directional wave spectra are extracted from the NOAA data for each of the three sites and the wave direction PDF is estimated according to each of the methods-direct frequency approach (Eq. 5) and Gaussian-kernel-smoothed (Eq. 7)-described in Section 3.1. The graphical results are presented in Figure 5 and show clearly how the Gaussian kernel smooths the wave direction PDF and spreads probability mass over a greater range of angles. For Maine this smoothing results in a much smaller peak probability density and a range of possible wave directions from $60^{\circ}$ to $210^{\circ}$ rather than $90^{\circ}$ to $180^{\circ}$ for the direct frequency-based estimate. For MA the differences between the two estimates of the wave direction PDF are less pronounced, and for Delaware smoothing removes two strong peaks in probability mass at $330^{\circ}$ and $150^{\circ}$. The results section shows how the directional IWWA depends on the choice of model for the wave direction PDF. It is also interesting that all the PDFs are centered on the range from $120^{\circ}$ to $150^{\circ}$. This is mainly caused by the similarity of their location off the coast as illustrated in Figure 5 in which the coast lies to the west or northwest and open ocean to the east, south, and southeast.

It is important to note that estimation of the wave direction PDF as is done here is worthwhile when a unidirectional wave field is used in analysis. If analysis is based on a multidirectional wave field simulated to correspond to the full directional spectrum then an alternative approach to directional analysis would directly treat the parameters of the directional spectrum as random parameters. Unidirectional wave field simulation is, however, industry standard at this time and 
furthermore tools for simulating multi-directional wave field are not integrated into standard
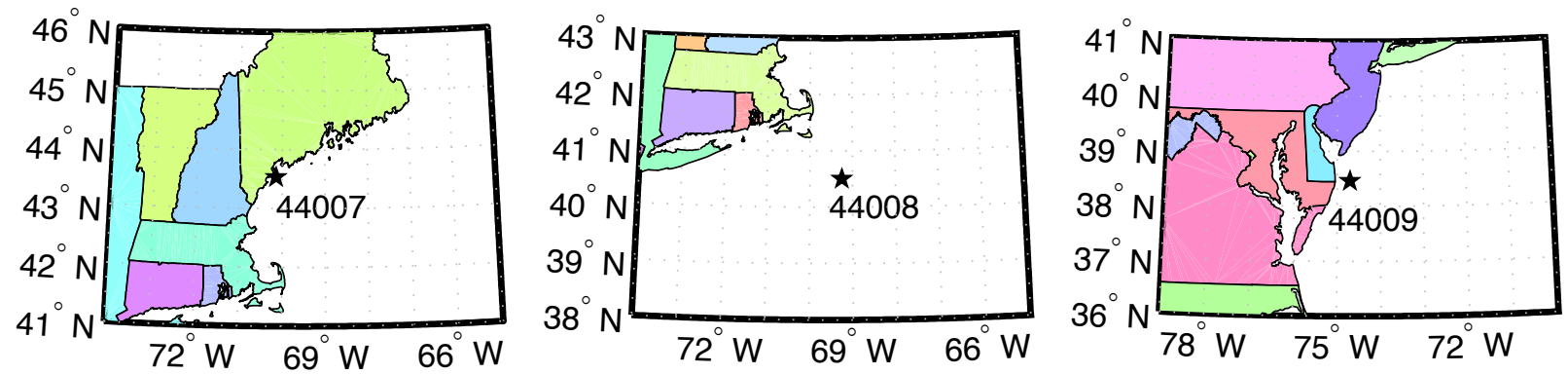

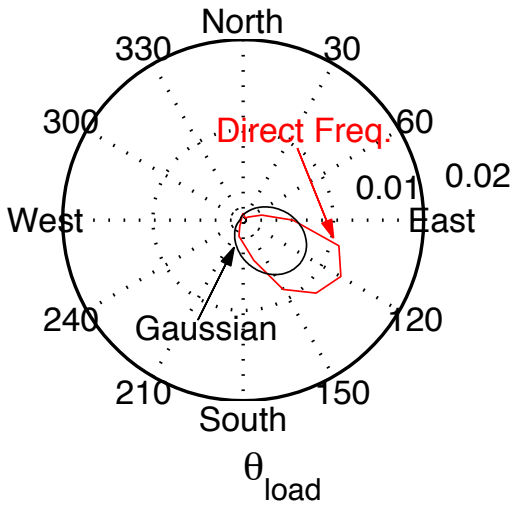

(a) Station 44007 (ME)

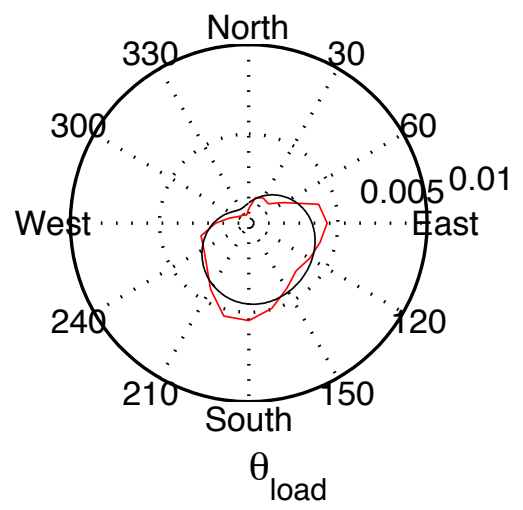

(b) Station 44008 (MA)

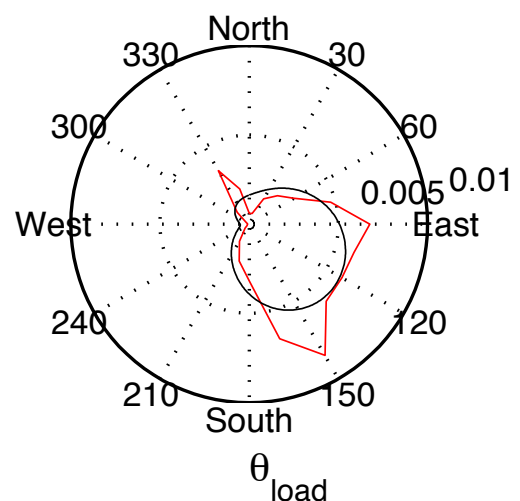

(c) Station 44009 (DE)
433

434

435

\section{6}

Figure 5 PDF of the most likely wave direction (direct frequency-based PDF plotted in red and Gaussian-kernel-smoothed PDF plotted in black)

\section{Results and Discussion}

\subsection{Jacket capacity and load direction}

As described in Section 4.1, the example jacket used for illustrative purposes here has four legs and rotational symmetry with a period of $90^{\circ}$ and is therefore non-axisymmetric. Figure 6(a) shows the MRP of the environmental conditions that cause failure of the jacket as a function of $\left|\theta_{\text {load }}-\theta_{\text {jacket }}\right|$ for the MA site. These results are extracted from the set of IWWAs shown in Figure 7. In Figure 7, $\gamma_{\max }$ is a scaling factor that indicates by how much the loading demands would need to be scaled to reach the ultimate capacity. When $\gamma_{\max }$ is smaller than 1 , structural ultimate capacity is less than the wind and wave load demand and hence the structure fails. Results are qualitatively similar for the other sites with only changes in the MRP values resulting from the different recurrences of hazard intensities at the different sites. The capacity of the structure is highly sensitive to the loading direction relative to the structural orientation with the lowest capacity occurring when the loading approaches at a $45^{\circ}$ angle to the structure. The difference in capacity from maximum to minimum is $-18 \%$ from $31,000 \mathrm{kN}$ to $25,300 \mathrm{kN}$ in terms of base shear, $-90 \%$ from 260,000 years to 25,000 years in terms of MRP, and $-5 \%$ from $16.2 \mathrm{~m}$ to 15.4 $\mathrm{m}$ in terms of significant wave height. The significant decrease of capacity at a $45^{\circ}$ loading angle 
452 originates from the fact that when loaded at $45^{\circ}$ the diamond-shaped plan of the structure is more susceptible to bending than the square plan at $0^{\circ}$.

454 Notice that a tiny difference of the significant wave height causes a big difference in failure MRP, 455 as shown in Figure 6(b). This is because at long MRPs very small increases in wave height and 456 wind speed correspond to very large increases in the MRP. This may be related to estimation of the hazard from continuous data rather than from a synthetic hurricane catalog containing thousands of years of data [41]. Use of such a catalog, while it would not affect the shape of the dependencies, would cause failure to occur at much smaller MRPs.
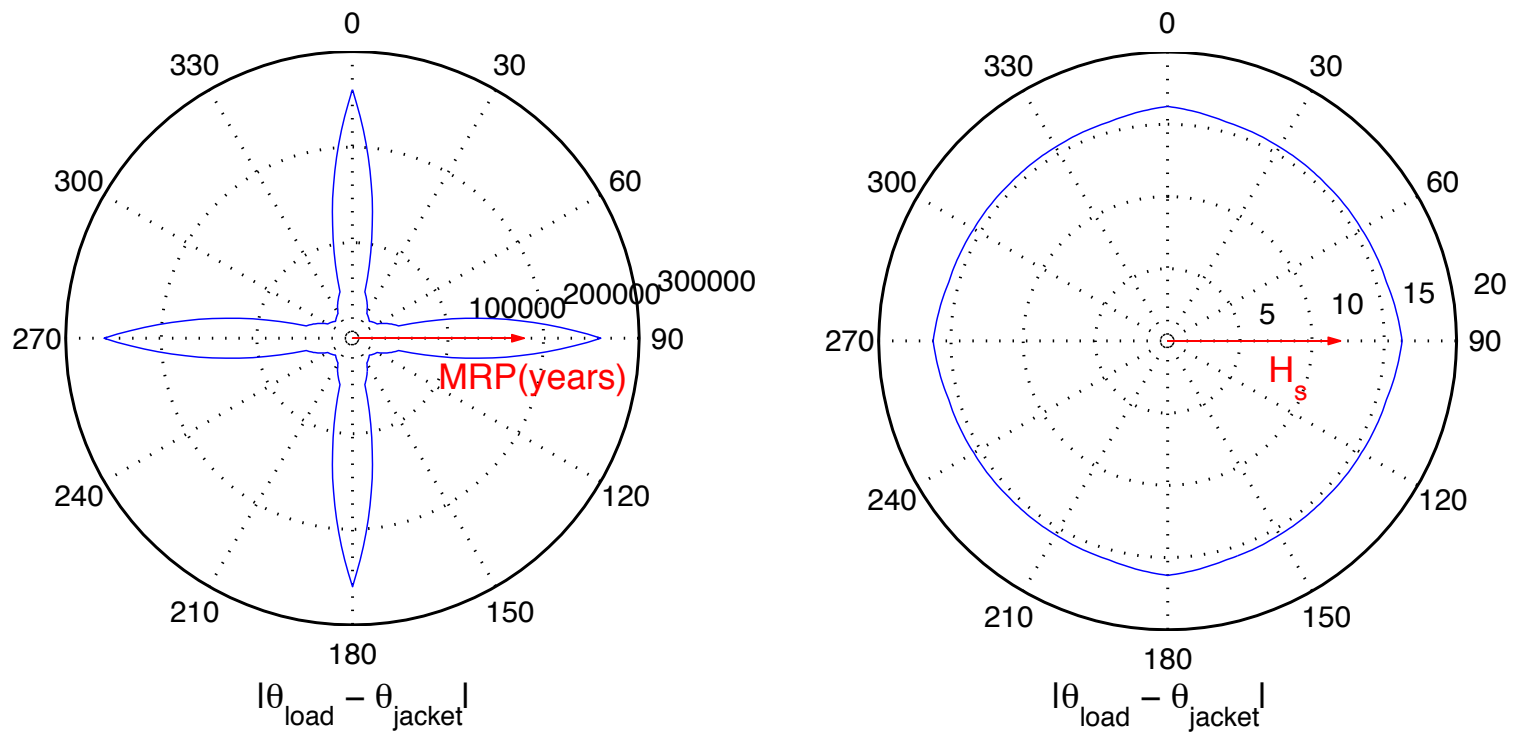

(a) Failure MRP for $\theta_{\text {jacket }}=0^{\circ}$ (year)

(b) Failure MRP corresponding $\mathrm{H}_{\mathrm{s}}$ for $\theta_{\text {jacket }}=0^{\circ}(\mathrm{m})$

Figure 6 Failure MRP (Unit: years) and corresponding $\mathrm{H}_{\mathrm{s}}$ (Unit: $\mathrm{m}$ ) of the environmental conditions that cause failure of the jacket as a function of $\left|\theta_{\text {load }}-\theta_{\text {jacket }}\right|$

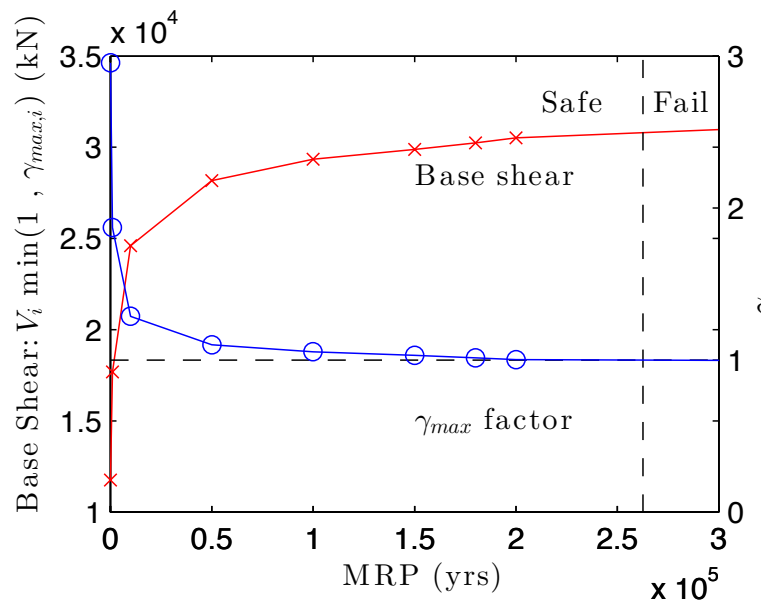

(a) $\left|\theta_{\text {load }}-\theta_{\text {jacket }}\right|=0^{\circ}$

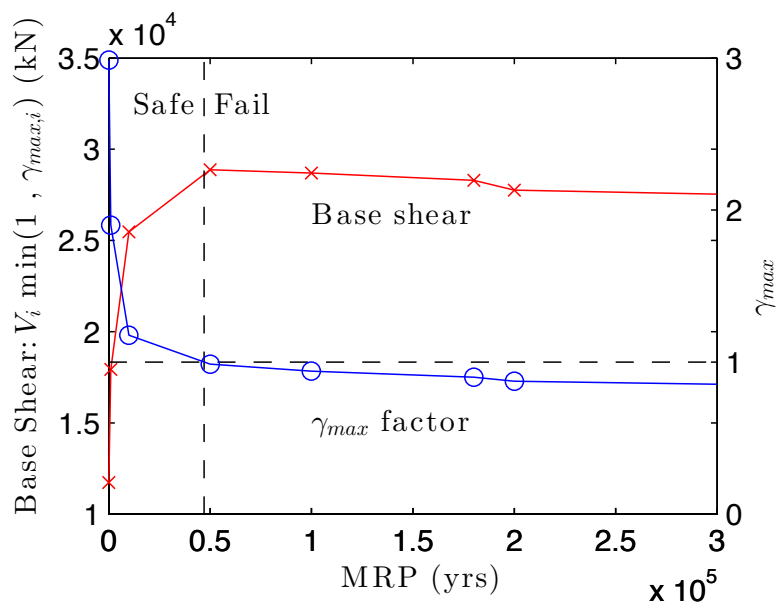

(b) $\left|\theta_{\text {load }}-\theta_{\text {jacket }}\right|=15^{\circ}$ 


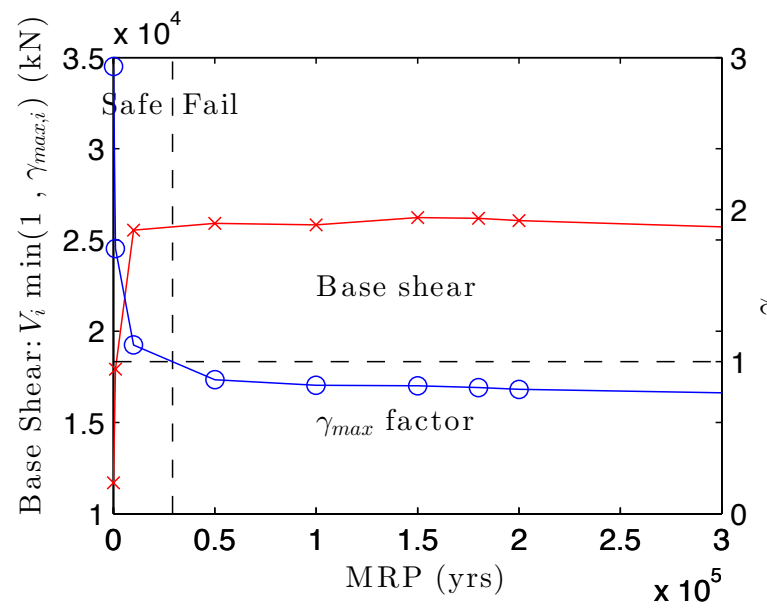

(c) $\left|\theta_{\text {load }}-\theta_{\text {jacket }}\right|=30^{\circ}$

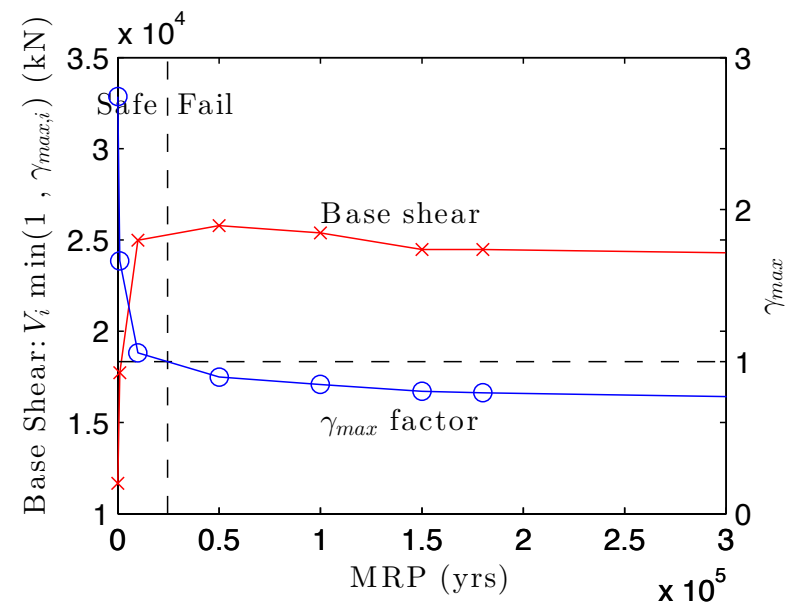

(d) $\left|\theta_{\text {load }}-\theta_{\text {jacket }}\right|=45^{\circ}$
466

467

Figure 7 Structural IWWA curves of the jacket under directional extreme wind and wave loading for $\left|\theta_{\text {load }}-\theta_{\text {jacket }}\right|=0,15,30$ and $45 \mathrm{deg}$.
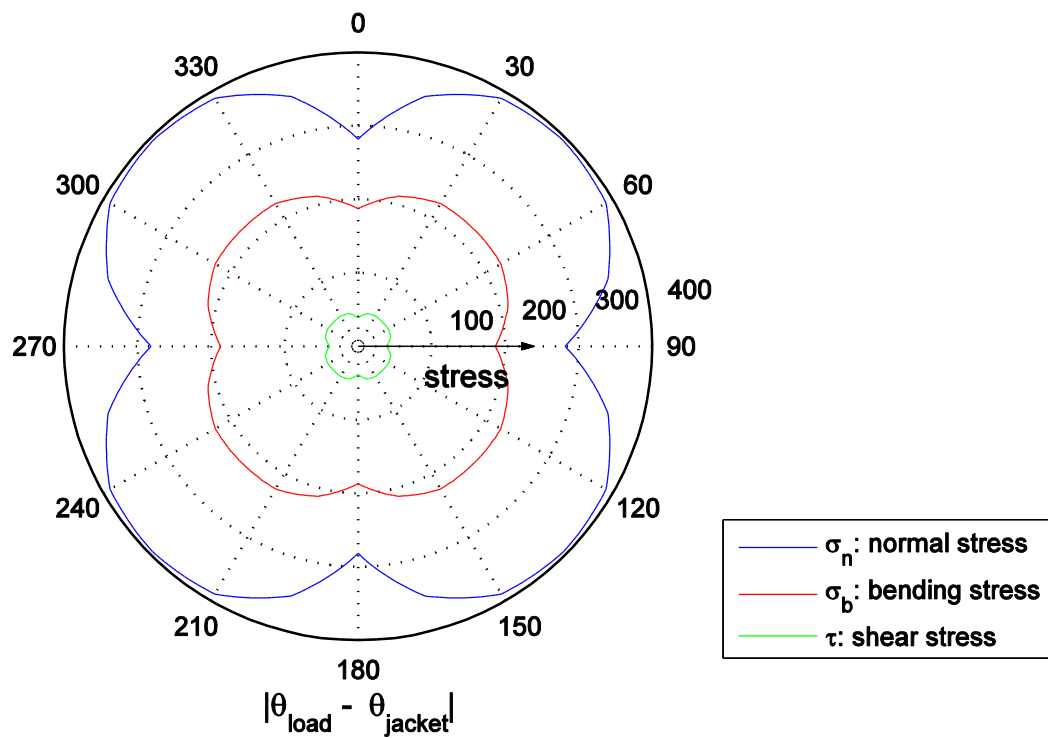

$\left|\theta_{\text {load }}-\theta_{\text {jacket }}\right|$

Figure 8 Absolute peak stresses (normal stress, bending stress and shear stress) in the bottom cross-section of the jacket leg at the downwind corner (MPa)

In order to better explain the dependence of the structural response on directionality, stresses are calculated for the bottom cross-section of the jacket leg at the downwind corner of the structure. This location is frequently the site of the formation of a fully developed mechanism in the pushover analysis. Figure 8 shows axial, bending and shear stresses, calculated for 10,000 year wind and wave conditions with gravity loads as a function of $\mid \theta_{\text {load }}$ - $\theta_{\text {jacket }} \mid$. In this figure, the highest stresses occur when the loading approaches at a $45^{\circ}$ angle to the structure. This result corresponds to the decreased capacity at this orientation and the increased likelihood of plastic hinge formation. 


\subsection{Effect of load directionality}

480 To investigate the effect of load directionality on the performance and reliability of the jacket structure, an example is now presented in which the jacket is assumed to be oriented with $\theta_{\text {jacket }}=$ $0^{\circ}$, which means that the jacket has one of its sides facing north. For each of the three sites the product $f_{\theta_{\text {load }}}\left(\theta_{\text {load }}\right) \times P_{f}\left(\theta_{\text {load }} \mid \theta_{\text {jacket }}=0\right)$ has been calculated and is shown in Figure 9 . In this expression $P_{f}\left(\theta_{\text {load }} \mid \theta_{\text {jacket }}=0\right)=1 / \mathrm{MRP}_{\text {fail }}$ is the conditional annual failure probability for the case where $\theta_{\text {jacket }}=0^{\circ}$ and the load is coming from a direction defined by $\theta_{\text {load. }}$. This conditional probability is probability density to the calculation of the aggregate probability of failure for a given structural orientation at a given site.

Presentation of the conditional probability in Figure 9 allows one to see visually how the directional dependence of jacket capacity and the directional wave PDF combine to render the performance of the jacket highly dependent on load direction. When the jacket orientates to the true north $\left(\theta_{\text {jacket }}=0^{\circ}\right.$ ), the Figure 9 (a-c) illustrates that waves coming from the diagonal directions of the jacket $\left(45^{\circ}, 135^{\circ}, 225^{\circ}, 315^{\circ}\right)$ are relatively more dangerous to the structure but that the strong peak of the directional wave PDF renders one of those directions (in this case $135^{\circ}$ for all three sites) the most critical.

495

496

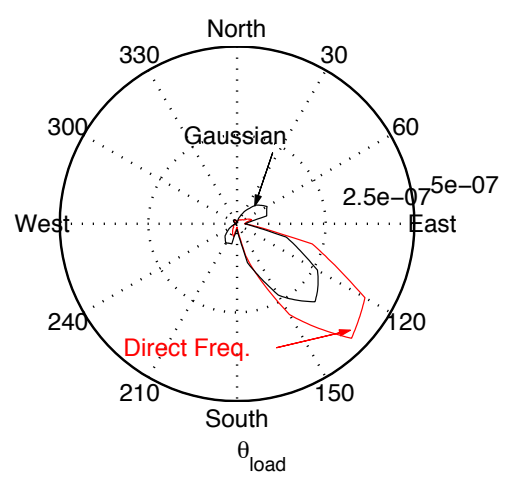

(a) Station 44007 (ME)

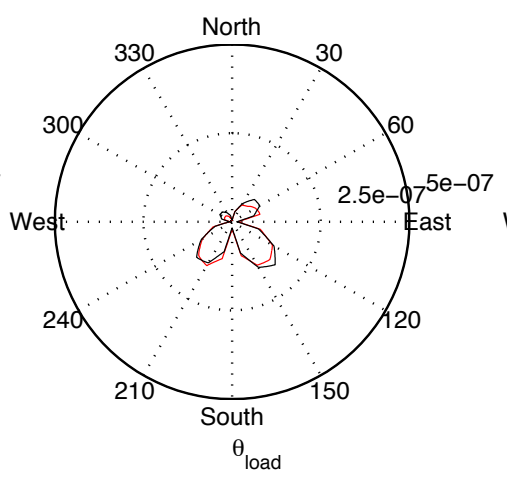

(b) Station 44008 (MA)

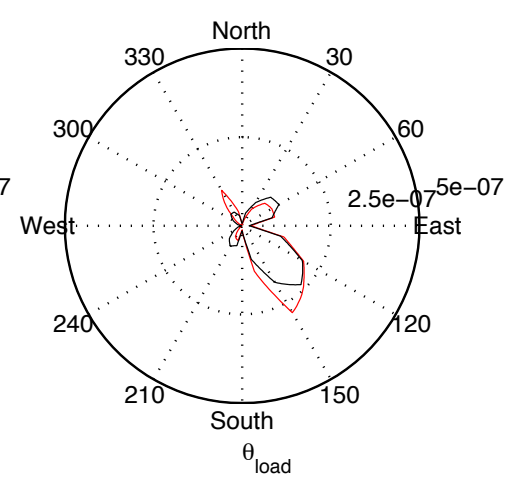

(c) Station 44009 (DE)

Figure 9 Conditional failure functions $f_{\theta_{\text {load }}}\left(\theta_{\text {load }}\right) \times P_{f}\left(\theta_{\text {load }} \mid \theta_{\text {jacket }}=0\right)$ as a function of $\theta_{\text {load }}$ at three different sites (results based on the direct frequency $f_{\theta_{\text {load }}}\left(\theta_{\text {load }}\right)$ is plotted in red and results on Gaussian-kernel-smoothed $f_{\theta_{\text {load }}}\left(\theta_{\text {load }}\right)$ plotted in black)

\subsection{Effect of structural orientation}

The final stage of a directional IWWA is to compute the aggregate probability of failure for a range of structural orientations at a given site to determine the directional dependence of the annual probability of failure. The directional failure probability in one year is calculated by Eq. for each of the two proposed models for the directional wave PDF. In general the results can be summarized as follows: 
1. Structural orientation has a modest effect on probability of failure for the sites investigated, with the greatest effect being approximately $20 \%$ for the Maine site. The optimized orientation of the jacket is $30^{\circ}$ for ME, $0^{\circ}$ for MA and $68^{\circ}$ for DE.

2. Implementation of the Gaussian kernel smoothed wave direction PDF dramatically reduces the predicted dependence of failure probability on structural orientation, rendering the failure probability essentially independent of structural orientation. The reason for this is that implementation of the Gaussian kernel essentially spreads the range of likely wave directions by $\pm 70^{\circ}$ and simultaneously lowers the peak of the wave direction distribution. The Gaussian kernel approach, however, should be considered less conservative than the direct frequency approach since it delivers a consistent failure probability that is lower than the maximum failure probability obtained from the direct frequency-based approach. In order to determine which model better approximates wave directionality in actual sea states, data would have to be available that measured the height and direction of each wave in, for example, a one hour sample of a sea state. Such data is not currently available.

3. Taking the structural orientation associated with minimum failure probability to be the optimal structural orientation, the three sites have dramatically different optimal orientations. These failure probabilities (Figure 10) are not readily discernible from Figure 9 since that figure shows the failure function only for $\theta_{\text {jacket }}=0$. Each point in Figure 10 results from integrating a function such as that shown in Figure 9 developed for a given structural orientation.
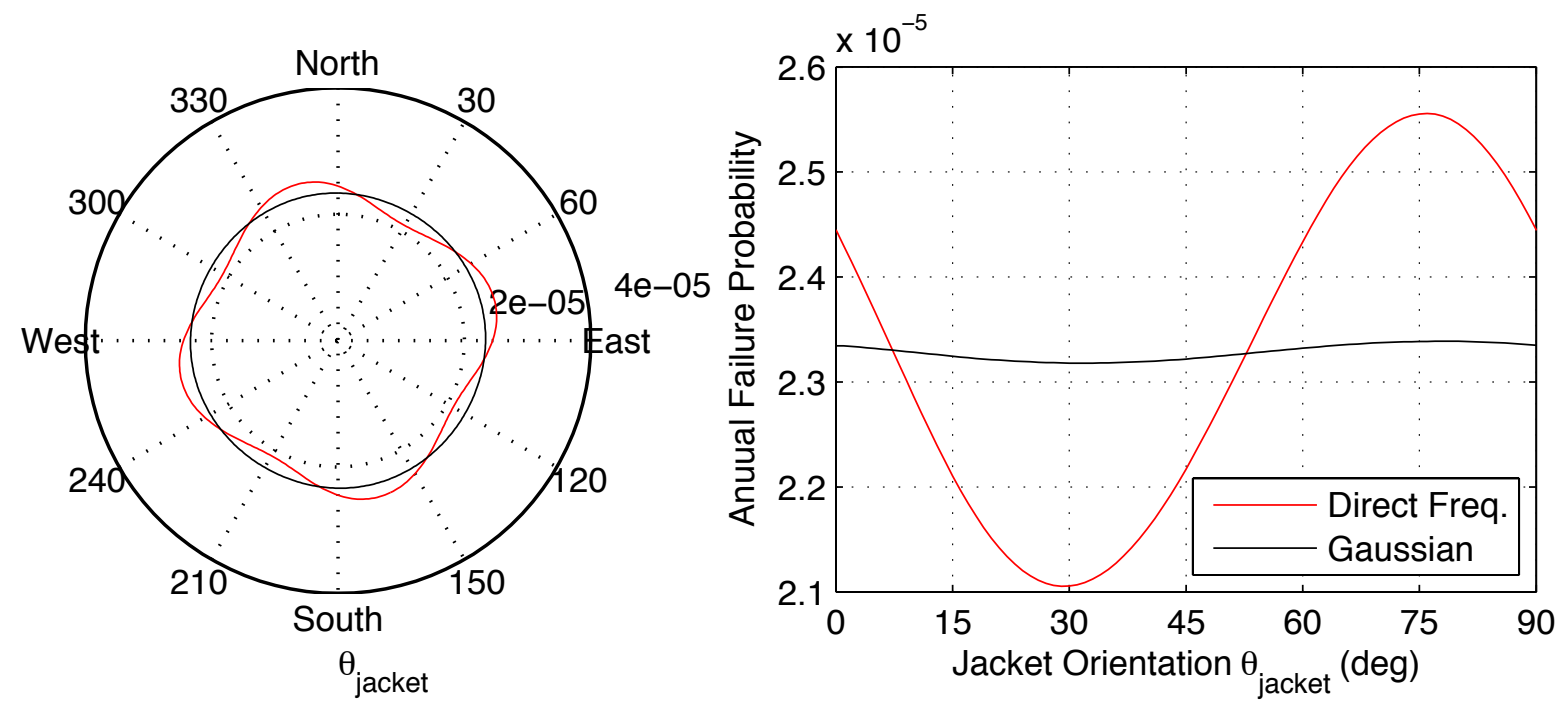

(a) Station 44007 (ME) 


\section{6}
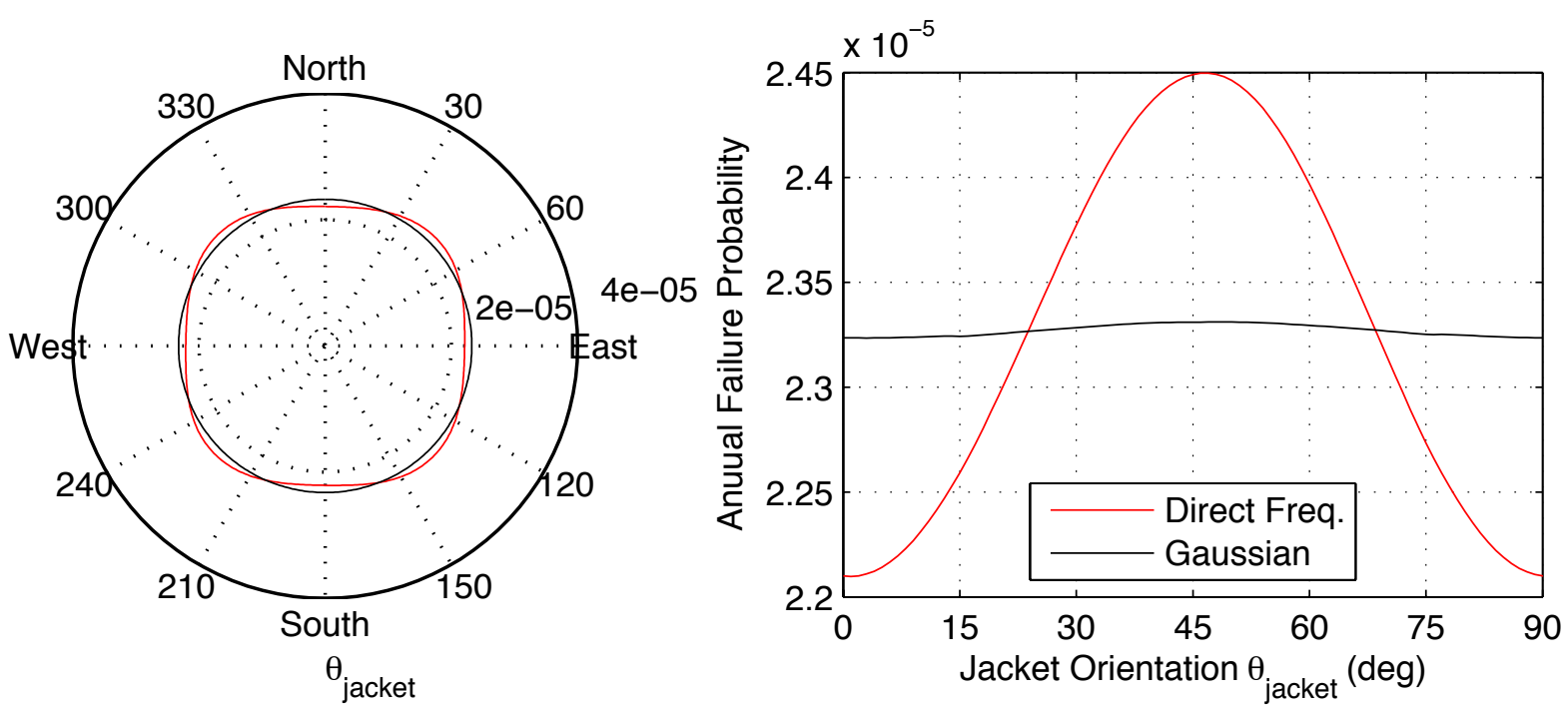

(b) Station 44008 (MA)
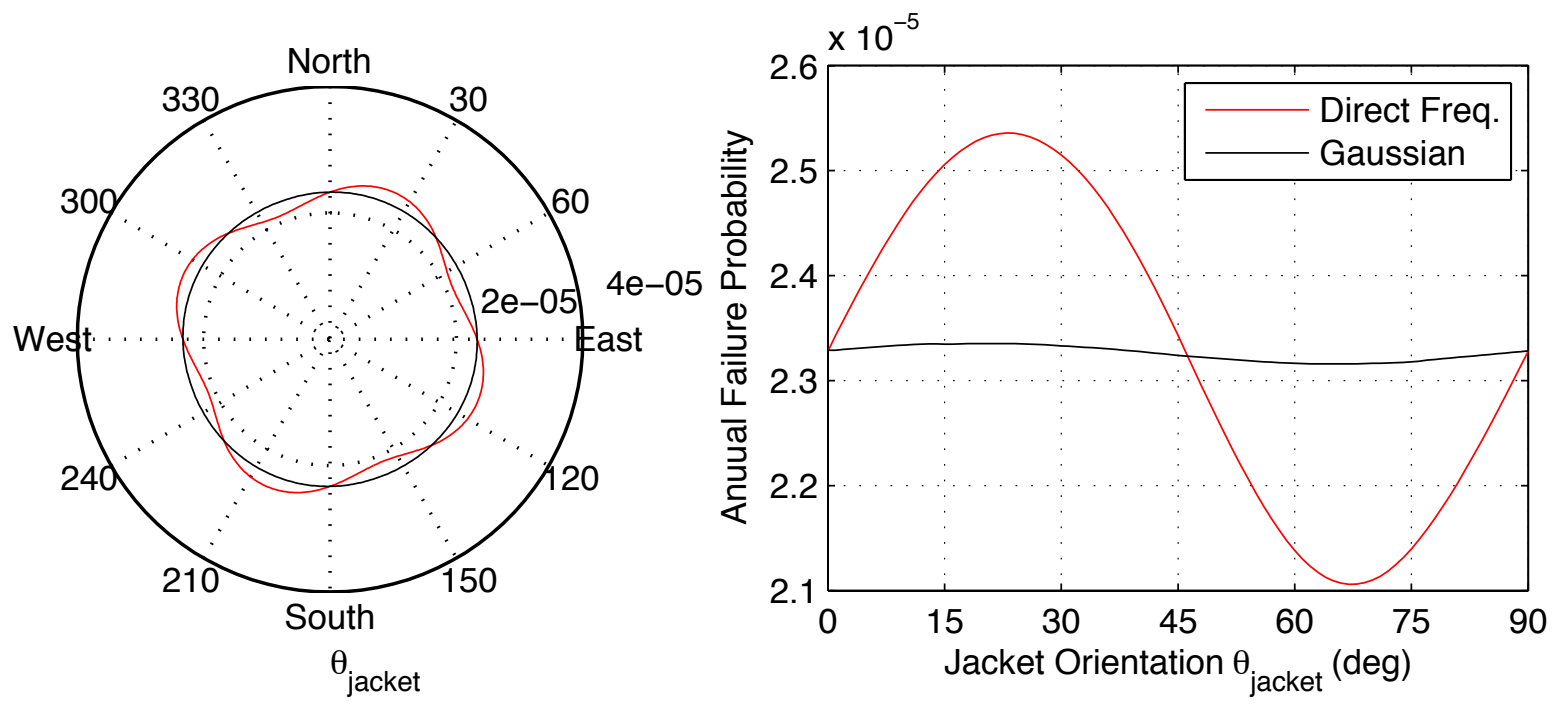

(c) Station 44009 (DE)

Figure 10 Overall $P_{f, 1}$ as a function of structural orientation $\theta_{\text {jacket }}\left(P_{f, 1}\right.$ obtained by the direct frequency-based $f_{\theta_{\text {load }}}\left(\theta_{\text {load }}\right)$ is plotted in red and $P_{f, I}$ by Gaussian-kernel-smoothed $f_{\theta_{\text {load }}}\left(\theta_{\text {load }}\right)$ plotted in black)

\section{Conclusion}

This paper has introduced an approach to assess the structural safety of jacket-supported OWTs under directionally dependent extreme environmental loads. The approach advances the Incremental Wind-Wave Analysis (IWWA) [22] of OWT jackets by including loading direction and jacket orientation in the framework. The novel approach allows structural safety assessment of jacket-type OWTs subjected to directional wind and wave load patterns corresponding to increasing mean return periods (MRPs). Two probability modeling approaches for measuring dominant wave directions data are provided to supply directional probability information of 
wind-wave loading. For each combination of wind-wave direction and structural orientation, structural capacity is assessed by IWWA1 procedure [22], which neglects the correlation between wind and wave loads and assumes the independent wind and wave conditions at equivalent MRPs occur simultaneously. Those capacities, convolved with MRP models for loading intensity, yield direction-dependent structural reliabilities, and, when those reliabilities are convolved with the probability density function for loading direction, a total failure probability is obtained that accounts for wave directionality. Example analyses are conducted for a four-leg jacket-supported OWT inspired by European Union UpWind Project. Measured directional metocean conditions at three sites along the U.S. Atlantic coast are used to illustrate the approach. These calculations illustrate that structural capacity is sensitive to the loading direction relative to the structural orientation and can be significantly decreased when the loading approaches at a $45^{\circ}$ angle to the structure. When loaded in that direction the capacity is only $80 \%$ of that when loaded at $0^{\circ}$ angle. The estimation of the wave direction PDF is important when only a unidirectional wave field is available for analysis. A study of the effect of structural orientation shows that structural orientation has a modest effect on probability of failure, with the greatest effect being approximately $20 \%$. Implementation of the Gaussian-kernel-smoothed wave direction PDF reduces the predicted dependence of failure probability on structural orientation. The structural orientation can be designed to minimize the failure probability through an optimization according to directional metocean conditions.

\section{Acknowledgement}

This work was supported financially by the US National Science Foundation through Grants 1234560 and 1234656 and by the Massachusetts Clean Energy Center. 
[1] European Wind Energy Association. Pure Power-Wind energy targets for 2020 and 2030: EWEA; 2011.

[2] U. S. Department of Energy. 20\% wind energy by 2030: Increasing wind energys contribution to US electricity supply. Washington, D.C.2008.

[3] International Renewable Energy Agency. Renewable energy technologies: cost analysis series - wind power. IRENA; 2012.

[4] API R. Recommended Practice for Planning, Designing, and Constructing Fixed Offshore Platforms-Working Stress Design. API RP 2A-WSD 21st Edition. Washington, D.C.: API Publishing Services; 2005.

580 [5] Qian J, Wang X-x. Three-dimensional stochastic response of offshore towers to random sea waves. Computers \& Structures. 1992;43:385-90.

[6] Van Der Tempel J. Design of support structures for offshore wind turbines: Delft University of Technology; 2006.

[7] Kim E, Manuel L. A Framework for Hurricane Risk Assessment of Offshore Wind Farms. ASME 2012 31st International Conference on Ocean, Offshore and Arctic Engineering: American Society of Mechanical Engineers; 2012. p. 617-22.

[8] Dong W, Moan T, Gao Z. Long-term fatigue analysis of multi-planar tubular joints for jacket-type offshore wind turbine in time domain. Eng Struct. 2011;33:2002-14.

[9] Haver S. Effects of wave directionality and choice of wave spectrum on the extreme response of a deep water jacket. Mar Struct. 1991;4:503-31.

[10] Bea R, Xu T, Stear J, Ramos R. Wave forces on decks of offshore platforms. Journal of Waterway, Port, Coastal, and Ocean Engineering. 1999;125:136-44.

[11] Cruz AM, Krausmann E. Damage to offshore oil and gas facilities following hurricanes Katrina and Rita: an overview. Journal of Loss Prevention in the Process Industries. 2008;21:620-6.

598 [13] Philippe M, Babarit A, Ferrant P. Modes of response of an offshore wind turbine with

[12] Li P. Analysis and Design of Offshore Jacket Wind Turbine: Norwegian University of Science and Technology; 2010. directional wind and waves. Renew Energ. 2013;49:151-5.

[14] Mittendorf K, Kohlmeier M, Habbar A, Zielke W. Influence of irregular wave kinematics description on fatigue load analysis of offshore wind energy structures. Proceedings of DEWEK2006. p. 22-3.

[15] Gudmestad OT, Moe G. Hydrodynamic coefficients for calculation of hydrodynamic loads on offshore truss structures. Mar Struct. 1996;9:745-58.

[16] Sharma J, Dean R. Second-order directional seas and associated wave forces. Publication of: Offshore Technology Conference1979.

[17] Isaacson M, Sinha S. Directional Wave Effects on Large Offshore Structures. Journal of Waterway, Port, Coastal, and Ocean Engineering. 1986;112:482-97.

[18] Goda Y. A comparative review on the functional forms of directional wave spectrum. Coastal Engineering Journal. 1999;41:1-20.

[19] Moon IJ, Ginis I, Hara T, Tolman HL, Wright CW, Walsh EJ. Numerical simulation of sea surface directional wave spectra under hurricane wind forcing. J Phys Oceanogr. 2003;33:1680706.

[20] Forristall G. On the Use of Directional Wave Criteria. Journal of Waterway, Port, Coastal, and Ocean Engineering. 2004;130:272-5. 
616 [21] Jonathan P, Ewans K. The effect of directionality on extreme wave design criteria. Ocean 617 Engineering. 2007;34:1977-94.

618 [22] Wei K, Arwade SR, Myers AT. Incremental wind-wave analysis of the structural capacity 619 of offshore wind turbine support structures under extreme loading. Engineering Structures. $620 \quad 2014 ; 79: 58-69$.

621 [23] Vorpahl F, Popko W, Kaufer D. Description of a basic model of the" UpWind reference 622 jacket" for code comparison in the OC4 project under IEA Wind Annex XXX. Fraunhofer 623 Institute for Wind Energy and Energy System Technology (IWES). 2011;4:1-14.

624 [24] Jonkman JM, Butterfield S, Musial W, Scott G. Definition of a 5-MW reference wind 625 turbine for offshore system development. National Renewable Energy Laboratory Colorado; 6262009.

627 [25] International Electrotechnical Commission. Wind Turbines - Part 3: Design Requirements 628 for Offshore Wind Turbines, IEC 61400-3. 2009.

629 [26] Ditlevsen O. Stochastic model for joint wave and wind loads on offshore structures. 630 Structural Safety. 2002;24:139-63.

631 [27] Tarp-Johansen NJ. Extreme wind and wave loads site-specific combination of design rules. 632 Proceeding of Copenhagen Offshore Wind2005.

633 [28] Eckert-Gallup AC, Sallaberry CJ, Dallman AR, Neary VS. Modified Inverse First Order 634 Reliability Method (I-FORM) for Predicting Extreme Sea States. Sandia National Laboratories 635 (SNL-NM), Albuquerque, NM (United States); 2014.

636 [29] Valamanesh V, Myers AT, Arwade SR. Multivariate analysis of extreme metocean conditions for offshore wind turbines. Structural Safety. 2015;55:60-9. [30] Golafshani AA, Bagheri V, Ebrahimian H, Holmas T. Incremental wave analysis and its application to performance-based assessment of jacket platforms. J Constr Steel Res. 2011;67:1649-57.

[31] Young IR. Directional spectra of hurricane wind waves. Journal of Geophysical Research: Oceans. 2006;111:C08020.

[32] Hu K, Chen Q. Directional spectra of hurricane-generated waves in the Gulf of Mexico. Geophysical Research Letters. 2011;38:L19608.

[33] Chen QJ, Hu K, Kennedy A. Numerical modeling of observed hurricane waves. Coastal Engineering Proceedings. 2011;1:waves.30.

[34] Steele KE, Mettlach T. NDBC wave data-current and planned. Ocean Wave Measurement and Analysis (1993): ASCE; 1993. p. 198-207.

[35] Earle MD, Steele KE, Wang DWC. Use of advanced directional wave spectra analysis methods. Ocean Engineering. 1999;26:1421-34.

[36] Martins ES, Stedinger JR. Generalized maximum-likelihood generalized extreme-value quantile estimators for hydrologic data. Water Resour Res. 2000;36:737-44.

[37] Jonkman JM, Buhl Jr ML. FAST user's guide. Golden, CO: National Renewable Energy Laboratory; 2005.

[38] Det Norske Veritas. Modelling and analysis of marine operations. DNV-RP-H103: DNV; 2011.

[39] Borgman L. Computation of the ocean-wave forces on inclined cylinders. Transactions, American Geophysical Union. 1958;39:885-8.

660 and Structures, Inc.; 1997. 
661 [41] Jha A, Dolan D, Musial W, Smith C. On hurricane risk to offshore wind turbine in US 662 waters. Proceedings of the 2010 Offshore Technology Conference. Houston, TX2010. 